\title{
ELLIPTIC DIFFERENTIAL OPERATORS AND DIFFUSION PROCESSES
}

\author{
HEINZ BAUER
}

This article develops survey lectures for general mathematical audiences which the author delivered at the 27th Annual Meeting of the Australian Mathematical Society at the University of Queensland, 1983, and at the 10th Austrian Congress of Mathematicians in Innsbruck, 1981. The central theme of these lectures was the use of probabilistic methods in the study of linear elliptic-parabolic differential equations of second order.

The starting point will be an orientative discussion of the role of Brownian motion in classical potential theory. It will then be discussed that, given an ellipticparabolic differential operator $L$ of a certain type, there exists a uniquely determined diffusion process which is linked with $L$ formally in the same way in which Brownian motion is linked with the Laplace operator. The fundamental results of K. Itô, D.W.Stroock and S.R.S. Varadhan will be in the centre of this part of the paper. We will then proceed to the discussion of more refined problems of the same type for differentiable manifolds. A glimpse at stochastic Riemannian geometry will then close our tour d'horizon.

Received 26 March 1984. This paper is based on an invited lecture given at the 1983 Australian Mathematical Society Annual Meeting held at the University of Queensland in May 1983.

Copyright clearance Centre, Inc. Serial-fee code: 0004-9727/84 $\$ A 2.00+0.00$. 


\section{Laplace Operator and Brownian Motion}

on $\mathbb{R}^{P}, p \geqslant 1$, equipped with the Euclidean norm $\|$.$\| , we$ consider the Brownian semigroup $\left(g_{t}\right)_{t>0}$ where

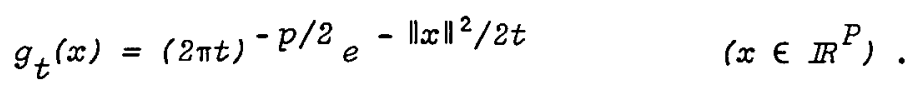

$\left(g_{t}\right)_{t>0}$ is a semigroup in the sense that $g_{s}{ }^{*} g_{t}=g_{s+t}$ holds for all $s, t>0$. It is advantageous to interpret the Brownian semigroup as a semigroup of positive linear operators, more precisely as a semigroup of kermels in the sense of measure theory (cf. [2], [16]). For this purpose we put

$$
P_{t} f=g_{t} * f \quad\left(f \in B^{b}\right) \text {, }
$$

where $B^{b}=B^{b}\left(\mathbb{R}^{p}\right)$ denotes the linear space of bounded, real-valued, Borel-measurable functions on $\mathbb{R}^{p}$. The semigroup property then appears in the form

$$
P_{s+t}=P_{s} P_{t}
$$$$
(s, t>0) \text {. }
$$

It is easily seen that $\left(P_{t}\right)_{t>0}$ is a Feller semigroup (or Feller-Dynkin semigroup in the terminology of Williams [23], p.115) which means that $\left(P_{t}\right)_{t>0} \quad$ is a semigroup of kernels having the following three properties:

$$
\begin{aligned}
& \left(F_{1}\right) \\
& P_{t}\left(C_{0}\right) \subset C_{0}, \\
& \left(\mathrm{~F}_{2}\right) \\
& \lim _{t \rightarrow 0}\left\|P_{t} f-f\right\|=0 \\
& \left(f \in c_{0}\right) \text {, } \\
& \left(\mathrm{F}_{3}\right) \\
& P_{t} 1=1 \text {. }
\end{aligned}
$$

Here $C_{0}=C_{o}\left(\mathbb{R}^{p}\right)$ denotes the space of continuous real-valued functions on $\mathbb{R}^{p}$ vanishing at infinity and $\|.$.$\| stands for the sup-norm.$

The semigroup $\left(P_{t}\right)_{t>0}$ is closely related to the Laplace operator 
$\Delta$. In fact, it is well known that $C_{c}^{2}$, the space of $C^{2}$-functions on $R^{p}$ with compact support, is contained in the domain of the infinitesimal generator of the Brownian semigroup and that on $C_{c}^{2}$ the infinitesimal generator coincides with $\frac{1}{2} \Delta$ (cf. [5]). So we have

$$
\lim _{t \rightarrow 0} \frac{p_{t} f-f}{t}=\frac{1}{2} \Delta f \quad\left(f \in C_{c}^{2}\right)
$$

(even in the sense of uniform convergence). From the semigroup property (3) the following integrated form of (4) follows:

$$
P_{t} f(x)=f(x)+\int_{0}^{t} P_{s}\left(\frac{1}{2} \Delta f\right)(x) d s \quad\left(f \in C_{c}^{2}\right)
$$

for all $x \in \mathbb{R}^{p}$.

When speaking about the Brownian semigroup $\left(g_{t}\right)_{t>0}$ or $\left(P_{t}\right)_{t>0}$, we have passed from the Laplace equation to the heat equation

$$
\frac{\partial u}{\partial t}-\frac{1}{2} \Delta u=0
$$

In fact, for $f \in B^{b}$, the function

$$
u(x, t)=P_{t} f(x)
$$

solves the heat equation on the half-space $\left.\mathbb{I R}^{p} \times\right] 0,+\infty l$. Consequently the property $\left(F_{2}\right)$ expresses that $u$ solves the Cauchy problem for $f \in C_{c}^{2}$ on this half-space. The function

$$
(x, t) \rightarrow g_{t}(x)
$$

given by (1) is nothing else than the fundomental solution of (6) with pole at the origin $(0,0)$.

We shall now pass to the probabilistic meaning of the Brownian semigroup. In the language of a physicist, this means that we replace the macroscopic observation level by the microscopic one. We introduce

$$
\Omega=C\left(\mathbb{R}_{+}, \mathbb{R}^{p}\right)
$$


the set of all continuous paths in $\mathbb{I K}^{p}$, i.e. of all continuous maps $\omega: \mathbb{R}_{+} \rightarrow \mathbb{I R}^{p}$ where $\mathbb{R}_{+}$is the closed half-line $10,+\infty[$. We introduce the position map for the time $t \in \mathbb{R}_{+}$

$$
\begin{aligned}
x_{t}: \Omega & \rightarrow \mathbb{R}^{p} \\
\omega & \rightarrow \omega(t)
\end{aligned}
$$

as well as the o-algebras

$$
F=\sigma\left(X_{s} ; s \in \mathbb{R}_{+}\right)
$$

and

$$
F_{t}=\sigma\left(X_{s}: s \in[0, t]\right)
$$

generated by all. $X_{s}$ respectively by all $X_{s}$ with $0 \leqslant s \leqslant t$. $F$ is the $\sigma$-algebra of all events (of interest to us); $F_{t}$ is the o-algebra of events up to time $t$.

on $F$ the Wiener measure $P$ is available. It is the only probability measure on $F$ with the following three properties:

$\left(\mathrm{w}_{1}\right) \quad P\left\{X_{0}=0\right\}=1$

$\left(w_{2}\right) \quad x_{t}-X_{s}$ is independent of $F_{s} \quad(s<t)$;

$\left(w_{3}\right)$ the distribution of the increment $X_{t}-X_{s}, s<t$, equals the $\mathrm{p}$-dimensional Lebesque measure

$\lambda^{p}$ with $g_{t-s}$ as density, i.e.

$P\left\{X_{t}-X_{s} \in B\right\}=\int_{B} g_{t-s} d \lambda^{p}$

for every Borel set $B \subset \mathbb{R}^{p}$.

Translation $\omega \rightarrow x+\omega$ yields a measurable bijection of $\Omega$ onto itself for every $x \in \mathbb{R}^{p}$. We denote by $P^{x}$ the image of $P$ under this bijection. While $P$ lives - according to $\left(W_{1}\right)$ - on the set of paths $\omega \in \Omega$ starting at the origin, $p^{x}$ lives on $\left\{X_{0}=x\right\}$ the set of 
paths $\omega \in \Omega$ starting at $x$. The family $\left(P^{x}\right)_{x \in \mathbb{R}} p$ of probability measures is called Brownian motion, it is the steering mechanism of a stochastic process describing the movement of a Brownian particle. It is important to observe that the family $\left(P^{x}\right)$ has the following properties :

$$
\begin{array}{ccr}
\left(\mathrm{D}_{1}\right) & P^{x}\left\{X_{0}=x\right\}=1 & \left(x \in \mathbb{R}^{p}\right) ; \\
\left(\mathrm{D}_{2}\right) & x \rightarrow P^{x}(A) \text { is Borel measurable } & (A \in F) ; \\
\left(\mathrm{D}_{3}\right) & E^{x}\left(f \circ X_{t} \mid F_{s}\right)=E^{X} s\left(f \circ X_{t-s}\right) & \begin{array}{c}
P^{x} \text {-almost surely } \\
\left(x \in \mathbb{R}^{p}, s<t, f \in B^{b}\right) .
\end{array}
\end{array}
$$

As usual, we denote by $E^{x}$ (resp. $E^{x}(. \mid$.$) ) the expectation (resp. the$ conditional expectation) with respect to $P^{x} \cdot\left(D_{3}\right)$ is the famous (weak) Markov property. It interrelates the different probability measures $P^{x}$ in such a way that the Brownian particle 'has no memory': not the full history of the particle counts for the prediction of its future; for the prediction, only the present position of the particle (at time $s$ ) is necessary. (For details, see for example [2].)

An arbitrary family $\left(P^{x}\right)_{x \in I R^{p}}$ of probability measures on $F$ with the above properties $\left(D_{1}\right)-\left(D_{3}\right)$ is called a diffusion (or diffusion process) on $\mathbb{R}^{p}$. So Brownian motion is a particular diffusion.

The Brownian semigroup $\left(P_{t}\right)_{t>0}$ can be recovered from Brownian motion (cf. [2], p.399-401). One has

$$
P_{t} f(x)=E^{x}\left(f \circ X_{t}\right) \quad \text { for all } f \in B^{b} \text {, }
$$

$x \in \mathbb{R}^{p}, t>0$. For an arbitrary diffusion the equalities (11) can be used in order to define a family $\left(P_{t}\right)_{t>0}$ of operators on $B^{b} \cdot\left(P_{t}\right)_{t>0}$ turns out to be a semigroup of Markovian kernels on $\mathbb{R}^{p}$, hence satisfying $P_{t}{ }^{1}=1$ for all $t>0 . \quad\left(P_{t}\right)_{t>0}$ is called the transition semigroup of the given diffusion. This semigroup is not a Feller 
semigroup, in general. Property $\left(F_{1}\right)$ is missing. Only a weakened form of $\left(F_{2}\right)$ - however equivalent to $\left(F_{2}\right)$ in the presence of $\left(F_{1}\right)$ (cf. [23], p.115) - is available, namely $\left(P_{t} f\right)_{t>0}$ converges pointwise to $f$ as $t \rightarrow 0$ (even for continous bounded functions $f$ ). Property $\left(\mathrm{F}_{3}\right.$ ) remains valid.

The great advantage of interpreting the Brownian semigroup as the transition semigroup of a diffusion process, namely of Brownian motion, lies in the well-known possibility of a probabilistic, intuitively simple approach to the main results of classical potential theory (cf. [6] and [3]). The price that one has to pay for this advantage is not negligible since one has to pass from the Laplace operator to the fundamental solution of the heat equation in order to arrive at the Brownian semigroup. For more general elliptic or even elliptic-parabolic differential operators a remarkable amount of hard analysis can be expected in order to arrive (eventually) at similar probabilistic approaches to potential theoretic problems. The theory of harmonic spaces develops a general machinery which allows to pursue this kind of an approach (cf. [3], [4], [7]).

In what follows we intend to discuss procedures which, for certain elliptic-parabolic differential operators $L$, will allow a probabilistic approach to the corresponding potential theory by going straight-away from $L$ to an associated diffusion.

2. Elliptic-parabolic differential operators and associated diffusions We will consider a linear differential operator $L$ defined on all of $\mathbb{I}^{p}$ of the form

$$
L=\frac{1}{2} \sum_{i, j=1}^{p} a_{i j}(x) \frac{\partial^{2}}{\partial x_{i} \partial x_{j}}+\sum_{i=1}^{p} b_{i}(x) \frac{\partial}{\partial x_{i}}
$$


which satisfies the following assumptions: all coefficients

$$
x \rightarrow a_{i j}(x) \text { and } x \rightarrow b_{i}(x)
$$

are bounded and continuous real-valued functions on $\mathbb{R}^{p}$. L is semielliptic (or elliptic-parabolic) in the sense that the matrix

$$
a(x)=\left(a_{i j}(x)\right)
$$

is symmetric and positive semi-definite for all $x \in \mathbb{R}^{p}$. The operator $L$ is called elliptic if $a(x)$ is even positive definite for all $x \in \mathbb{R}^{p}$ A diffusion $\left(P^{x}\right)_{x \in R^{p}}$ on $\mathbb{I R}^{p}$ will be called associated to $L$ if its transition semigroup $\left(P_{t}\right)_{t>0}$ satisfies the same condition as Brownian motion does for the special operator $L=\frac{1}{2} \Delta$ (see (5)), namely:

$$
P_{t} f(x)=f(x)+\int_{o}^{t} P_{s}(L f)(x) d s \quad\left(f \in C_{c}^{2}, x \in \mathbb{R}^{p}\right) .
$$

According to the definition (11) of the transition semigroup and according to $\left(D_{1}\right)$, this can be rewritten in the form

$$
E^{x}\left(H_{t}^{f}\right)=0
$$$$
\text { ( } f \in C_{c}^{2}, x \in \mathbb{R}^{p} \text { ), }
$$

where

$$
H_{t}^{f}=f \circ X_{t}-f \circ X_{0}-\int_{0}^{t} L f \circ X_{s} d s
$$

is defined on the set $\Omega$.

The probability measures $P^{x}$ of a diffusion associated to $L$ are interrelated by means of the Markov property $\left(\mathrm{D}_{3}\right)$ in a complicated way. The family $\left(H_{t}^{f}\right)_{t \geqslant 0}$ of real random variables leads to the possibility of detangling these measures with the intention to prove the existence of diffusions associated to $L$ later on. This possibility is due to the following fact which can be easily proved: $\left(H_{t}^{f}\right)_{t \geqslant 0}$ is a martingale with respect to each probability measure $P^{x}$ of a given diffusion associated with $L$. So, by definition, 


$$
E^{x}\left(H_{t}^{f} \mid F_{s}\right)=H_{s}^{f} \quad P^{x} \text {-almost surely }
$$

whenever $o \leqslant s<t, x \in \mathbb{R}^{p}$ and $f \in C_{c}^{2}$.

This crucial observation leads to the following

DEFINITION. A probability measure $P$ on $F$ is called a solution of the martingale problem for $L$ and for a point $x \in \mathbb{R}^{p}$ if $\left(M_{1}\right) \quad\left(H_{t}^{f}\right)_{t \geqslant 0}$ is a martingale with respect to $\mathrm{P}$ ( $\left.f \in C_{c}^{2}\right)$ and if

$$
\left(\mathrm{M}_{2}\right) \quad P\left\{X_{0}=x\right\}=1 .
$$

For reasons of illustration let us return for a moment to the situation of $\$ 1$, namely to $L=\frac{1}{2} \Delta=\frac{1}{2} \frac{d^{2}}{d x^{2}}$ in dimension $p=1$. For the two functions $f=i d$ and $=i d^{2}$, that is $f(x)=x$ and $=x^{2}$, one obtains for any probability measure $P$ on $F$ satisfying $P\left\{X_{0}=0\right\}=1$ that $H_{t}^{i \vec{d}}$ and $H_{t}^{i d^{2}}$ coincide with

$$
X_{t} \text { and } X_{t}^{2}-t
$$

P-almost surely,

respectively. According to $\left(W_{1}\right)$ the (1-dimensional) Wiener measure is such a measure $P$. It is a classical result of P. Lévy (cf. [10], p.75) that $\left(X_{t}\right)_{t \geqslant 0}$ and $\left(X_{t}^{2}-t\right)_{t \geqslant 0}$ are martingales for a probability measure $P$ on $F$ satisfying $P\left\{X_{0}=0\right\}=1$ if and only if $P$ is the wiener measure. From this it can be derived that the Wiener measure is the only solution of the martingale problem for $x=0$ (given the operator $L=\frac{1}{2} \Delta$ in dimension $p=1$ ). The proof has to take care of the fact that the two functions $i d$ and $i d^{2}$ do not have a compact support.

It is the preceding illustration which motivates the following definition - now again in the general situation.

DEFINITION. The martingale problem (for the differential operator $L)$ is said to be well posed if, for every $x \in \mathbb{R}^{p}$, it has exactly one 
solution.

By means of compactness arguments involving probability measures, one is led to the first result of fundamental importance due to stroock and Varadhan [20], [21]. (For quick references see the presentation of Priourret [18] .)

PROPOSITION 1. Assume that the martingale problem for $L$ is well posed. Then the fomily of its solutions $\left(P^{x}\right)_{x \in \mathbb{R}} p$ is the only diffusion associated to L. Furthermore, the corresponding transition semigroup $\left(P_{t}\right)_{t>0}$ is a Eelzer semigroup.

One is thus led to the problem of deciding when the martingale problem is well posed. Essentially, two types of answers are known:

THEOREM 1. In each of the following two cases the martingale problem is well posed:

(I) the matrix field $x \rightarrow a(x)$ is of the form

$$
a(x)=\sigma(x) \sigma^{T}(x) \quad\left(x \in \mathbb{R}^{p}\right)
$$

where the matrix field $x \rightarrow \sigma(x)$ as well as the vector field $x \rightarrow\left(b_{1}(x), \ldots, b_{p}(x)\right)$ satisfy the global Lipschitz condition on $\mathbb{R}^{p}$. (Sv) The differential operator $L$ is elliptic.

condition (I) is due to $K$. Itô [11]. The proof uses the technique of stochastic differential equations. (SV) is due to stroock and varadhan [20], [21]. The proof makes essential use of the martingale formulation by means of an approximation of the situation (SV) by that of (I). Furthermore, it uses the so-called Cameron-Martin formula which allows the passage from the case where the drift, which by definition is the vector field

$$
x \rightarrow b(x)=\left(b_{1}(x), \ldots, b_{p}(x)\right),
$$

vanishes to the general case by means of a martingale argument. For the technical details the reader is referred to Priouret [18]. 
A Feller semigroup associated to $L$ can always be interpreted as the transition semigroup of a diffusion (cf. [23]). This fact leads to a purely non-probabilistic version of Theorem 1 in connection with Proposition 1 .

COROLLARY. Under each of the assumptions (I) or (SV) there exists exactly one Feller semigroup $\left(P_{t}\right)_{t>0}$ for which condition (13) is satisfied.

No purely analytic proof of this result seems to be known. In conclusion we can say that by means of the martingale problem it is possible to pass directly from the differential operator $L$ to an associated diffusion process which, in addition, turns out to be uniquely determined by each of the conditions (I) and (SV).

\section{Diffusions on manifolds}

Up to now the coefficients of the differential operator $L$ were assumed to be bounded and continuous). The treatment of the continuous, but unbounded case leads in a natural way to the problem of studying diffusions associated to a semi-elliptic differential operator on a differentiable manifold. In fact, the natural idea of treating the unbounded case by restricting the differential operator $L$ to open subsets $U$ of $\mathbb{R}^{p}$ on which $L$ coincides with a differential operator $L_{U}$ on $\mathbb{R}^{p}$ satisfying the conditions of $\$ 2$, by restricting the diffusion associated to $L_{U}$ to the set $U$ in an appropriate way, and by glueing together the pieces can be carried out successfully (cf. [18]).

So let us pass immediately to the case of a $C^{\infty}$-manifold $M$ of dimension $p$. We assume that $M$ is connected and has a countable base. A cemi-elliptic (respectively elliptic) differential operator on $M$ will be a map

$$
L: C^{2}(M) \rightarrow C(M)
$$


which in each chart $x=\left(x_{1}, \ldots, x_{p}\right)$ can be written in the form

$$
L=\frac{1}{2} \sum_{i, j=1}^{p} a_{i j}(x) \frac{\partial^{2}}{\partial x_{i} \partial x_{j}}+\sum_{i=1}^{p} b_{i}(x) \frac{\partial}{\partial x_{i}}+c
$$

where the coefficients are continuous and the matrix $\left(a_{i j}(x)\right)$ is positive semi-definite (respectively definite) for all admissible points $x$. For reasons of simplicity we will assume $L 1=0$; that is, $c=0$ as we did before. Contributions to the general case where $c=L 1 \leqslant 0$ can be found in [1].

The probabilistic setting of $\$ 1$ has now to be modified (even for $M=\mathbb{R}^{p}$ ) in order to study a phenomenon which unbounded coefficients cause in general, namely the explosion phenomenon. It is due to a possibly finite lifetime of the moving particle described by a diffusion. In order to take care of this phenomenon we consider the one-point compactification $M_{\delta}=M U\{\delta\}$ of $M$ (with $\delta$ as an isolated point if $M$ is compact) and the space $C_{\delta}(M)$ of all continuous maps $\omega: \mathbb{R}_{+} \rightarrow M_{\delta}$ which respect $\delta$ in the sense of a "cimetary": $\omega\left(t_{0}\right)=\delta$ for some $t_{0} \in \mathbb{R _ { + }}$ implies $\omega(t)=\delta$ for all $t \geqslant t_{0}$. The number

$$
\zeta(\omega)=\inf \left\{t \in \mathbb{R _ { + }}: \omega(t)=\delta\right\}
$$

is then called the lifetime of the path $\omega \in C_{\delta}(M)$.

The appropriate probabilistic setting is now the following: we choose $\Omega=C_{\delta}(M)$ and define $\left(X_{t}\right)_{t \geqslant 0}, F$ and $\left(F_{t}{ }^{\prime} t \geqslant 0\right.$ formally in the same way as before. A diffusion on $M$ is then a family $\left(P^{x}\right)_{x \in M}$ of probability measures on $\Omega$ with the properties $\left(D_{1}\right)-\left(D_{3}\right)$ reformulated in the appropriate way. The only essential difference to $\$ 1$ is the definition of $B^{b}$ : this has to be now the space of all bounded Borelmeasurable functions on $M$ extended to $M_{\delta}$ by assigning the value 0 
to $\delta$. The definition (11) of the transition semigroup $\left(P_{t}\right)_{t \geqslant 0}$ yields then

$$
\left.P_{t} 1(x)=P^{x_{1 X}} \in M\right\}=P^{\left.x_{1 \zeta}>t\right\}} \quad(x \in M)
$$

and hence

$$
P_{t}^{1} \leqslant 1
$$

So $\left(P_{t}\right)_{t \geqslant 0}$ is now a semigroup of sub-Markov kemels on $M$. All $P_{t}$ are Markov kernels on $M$, that is they satisfy

$$
P_{t} 1(x)=1 \quad\left(x \in M, t \in \mathbb{R}_{+}\right)
$$

if and only if

$$
\zeta=+\infty \quad \mathrm{P}^{x} \text {-almost surely }
$$

for all $x \in M$. For $M=\mathbb{R}^{p}$ this corresponds to the situation which we studied before. We do not encounter the phenomenon of an explosion there.

As before, one can study diffusions associated to $L$ and the martingale problem. Via localization and the technique of glueing pieces together, one is then led to (cf. [18], p.111).

THEOREM 2. There exists exactly one diffusion process on $M$ associated to $L$ if $L$ satisfies one of the following two conditions:

(I') $L$ has coefficients in $C^{2}(M)$

(S'v') $L$ is elliptic.

It is important to observe that both conditions are chart invariant. For $M=I R^{P}, C^{2}$-coefficients of $L$ imply that condition (I) is locally satisfied. The matrix field $a$ is then of the form $a(x)=\sigma(x) \sigma^{T}(x), x \in \mathbb{R}^{p}$, where the new matrix field $x \rightarrow \sigma(x)$ is locally Lipschitz (cf. [18], p.82).

In the situation of Theorem 2 the unique diffusion associated with $L$ has a transition semigroup $\left(P_{t}\right)_{t>0}$ which is not anymore uniquely determined by the one condition 


$$
P_{t} f=f+\int_{0}^{t} P_{s}(L f) d s
$$

However, (20) determines $\left(P_{t}\right)_{t>0}$ uniquely if there is no explosion (cf. [1]). Furthermore, $\left(P_{t}\right)_{t>0}$ is not a Feller semigroup in general, even if there is no explosion. So it is natural to ask, when there is no explosion and when the transition semigroup is of Feller type.

Several answers to these questions are known. We restrict our discussion to two particular situations in which interesting answers are available.

The first situation is that of $M=\mathbb{R}^{p}$. Several conditions are known which assure an infinite lifetime of the diffusion. One of the simplest and most important is the following (cf. [21], p. 255 for further details).

PROPOSITION 2. There is no explosion if the differential operator $L$ is elliptic and if for some constant $K>0$

$$
\|a(x)\| \leqslant K\left(1+\|x\|^{2}\right)
$$

and

$$
<x, b(x)>\leqslant K\left(1+\|x\|^{2}\right)
$$

hold for all $x \in \mathbb{R}^{p}$.

Similar, but somewhat stronger growth conditions are necessary to assure, in addition, that the transition semigroup of the diffusion associated to $L$ is a Feller semigroup. Condition (22) has to be replaced by

$$
|<x, b(x)>| \leqslant K\left(1+\|x\|^{2}\right)
$$

also a stronger version of $(21)$ is needed. For details the reader is referred to Leha-Ritter [13]. Conditions close to being necessary and sufficient can be found in Azencott [1] .

The second situation concerns the case where $M$ is a Riemannian (C)-)manifold and where 


$$
I=\frac{1}{2} \Delta_{M}
$$

is - up to the factor $\frac{1}{2}$ - the Laplace-Beltromi operator $\Delta_{M}$ of $M$. The unique diffusion associated to $L$ is called Brownian motion on $M$. It can also be obtained by the global solutions of a stochastic differential equation (cf. $[24]$, p.46 and the acknowledgement mentioned there).

The manifold $M$ is called stochastically complete if Brownian motion on $M$ does not explode. The following result is due to Yau [25] who gave an analytical proof. For a probabilistic proof see Ichihara [9]

THEOREM 3. Every complete Riemannian manifold $M$ with Ricci curvature bounded from below is stochastically complete. Furthermore, the transition semigroup of Brownian motion on $M$ is then a Felzer semigroup.

Here completeness of $M$ refers to metric completeness with respect to the global metric derived from the Riemannian metric. The curvature condition is fulfilled if the sectional curvature of $M$ is bounded from below. Related results have been obtained by Azencott [1], DebiardGaveau-Mazet [8] and Pinsky [17] .

\section{An outlook on stochastic Riemannian geometry}

In the preceding situation ( $M$ Riemannian manifold and $L=\frac{1}{2} \Delta_{M}$ ) the transition semigroup $\left(P_{t}\right)_{t>0}$ of Brownian motion on $M$ has a density $p_{t}(x, y)$ with respect to the natural measure $\lambda_{M}$ on $M$ which depends smoothly on $(t, x, y) \in] 0,+\infty[\times M \times M$ (cf. [1], [15]). So we have for all $f \in B^{b}$

$$
P_{t} f(x)=\int p_{t}(x, y) f(y) \lambda_{M}(d y) \quad(t>0) .
$$

For fixed $y \in M$ the function $(t, x) \rightarrow p_{t}(x, y)$ is the minimal fundamental solution of the heat equation 


$$
\frac{\partial}{\partial t}-\frac{1}{2} \Delta_{M}=0
$$

on $] 0,+\infty\left[\times M\right.$ with pole at $(0, y)$. For $M=\mathbb{R}^{p}$ we are then back in the framework of $\$ 1$ where

$$
p_{t}(x, y)=g_{t}(x-y)
$$

For the study of geometric properties of $M$ it is important to know estimates for $p_{t}$. The so-called comparison method of Malliavin [14] and his school uses Brownian paths and stochastic differential equations to obtain such estimates. We close this article with one remarkable result due to Debiard-Gaveau-Mazet [8] .

For fixed dimension $p$, let $M_{a}$ denote the canonical Riemannian manifold of constant sectional curvature a (and dimension $p$ ). Two geodesic balls with centres $x_{0} \in M$ and $x_{0}^{a} \in M_{a}$ of the same radius $\rho>0$ can be identified by means of the normal charts and a linear isometry of the tangent spaces at $x_{0}$ and $x_{0^{\prime}}^{a}$ for $\rho$ sufficiently small (cf. [14], p.38). In the case of a Hadamard manifold even global identification of $M$ and $M_{a}$ is possible due to the Cartan-Hadamardtheorem. We recall that $M$ is called a Hadomard manifold [22] if $M$ is (metrically) complete, simply connected and of sectional curvature $K \leqslant 0$.

Each manifold $M_{a}$ is such a Hadamard manifold provided that $a \leqslant 0$. The corresponding densities $p_{t}^{a}$ are known. They can be calculated by means of recursion formulas (cf. [8], p.397). In the case $a=0$ we have $M_{0}=\mathbb{R}^{p}$ and hence $p_{t}^{o}(x, y)=g_{t}(x-y)$. Also in the general case $p_{t}^{a}(x, y)$ depends on $x, y$ only via the distance $r=d(x, y)$ in the global metric.

The announced result of Debiard-Gaveau-Mazet [8] is now the following. 
THEOREM 4. Let $M$ be a Hadamard manifold which is stochastically complete. Suppose that its sectional curvature $K$ is bounded from above by a constant $a \leqslant 0$ or from below by a constant $b$. Then (after global identification of $M$ and $M_{a}$ ' one has

$$
p_{t}(x, y) \leqslant p_{t}^{a}(x, y) \quad(x, y \in M)
$$

and

$$
p_{t}^{b}(x, y) \leqslant p_{t}(x, y) \quad(x, y \in M)
$$

respectively

In particular, one always has

$$
p_{t}(x, y) \leqslant\left(\frac{1}{2 \pi t}\right)^{p / 2} e^{-r^{2} / 2 t} \quad \text { with } r=d(x, y)
$$

since $p_{t}^{o}(x, y)=g_{t}(x-y)$. It should be observed that, according to Theorem 3, lower boundeaness of $K$ implies stochastical completeness of M.

The results of Theorem 4 can be used in order to obtain comparison results about Green functions and the first eigenvalue of the differential operator $-\Delta_{M}$ (cf. [8]).

What is remarkable in the proofs of these results is the constant use of Brownian paths. They thus become the object of differentialgeometric studies. This is due to the fact that the density $p_{t}$ can be written in the form

$$
\begin{aligned}
p_{t}(x, y) & =\lim _{\varepsilon \rightarrow 0} \frac{1}{\lambda_{M}\left(B_{\varepsilon}(y)\right)} \mathrm{P}_{t} \mathrm{I}_{\mathrm{B}_{\varepsilon}(y)}(x) \\
& =\lim _{\varepsilon \rightarrow 0} \frac{1}{\lambda_{M}\left(B_{\varepsilon}(y)\right)} \mathrm{P}^{\left.x_{\left\{X_{t}\right.} \in B_{\varepsilon}(y)\right\}}
\end{aligned}
$$

where $B_{\varepsilon}(y)$ denotes the geodesic ball of radius $\varepsilon>0$ with centre $y$. Here again, we encounter the general message of this lecture: probabilistic methods, above all the theory of stochastic processes, allow deep insight in purely analytic problems. 
References

[1] R. Azencott, "Behaviour of diffusion semi-groups at infinity", Bulz. Soc. Math. France 102, (1974) 193-240.

[2] H. Bauer, Probability theory and elements of measure theory. (Academic Press, London-NewYork-Toronto-Sydney-San Francisco (1981).)

[3] H. Bauer, Harmonic spaces and associated Markov processes. In: Potential Theory, (Centro Internazionale Matematico Estivo (C.I.M.E.), Stresa 1969. Edit. Cremonese, Rome (1970)) .

[4] H. Bauer, Harmonische Rärme. (Jahrbuch Ubberblicke Mathematik 1981, 9-35. Bibliographisches Institut, Mannheim (1981)J

[5] C. Berg and G. Forst, Potential theory on locally compact abelian groups. (Ergebnisse der Math. 87 Springer-Verlag, BerlinHeidelberg-New York (1975).)

[6] K.L. Chung, Lectures from Markov processes to Brownian motion. (Grundlehren d. math. Wiss. 249, Springer-Verlag, New YorkHeidelberg-Berlin (1982).)

[7] C. Constantinescu and A. Cornea, Potential theory on harmonic spaces. (Grundlehren d. math. Wiss. 158, Springer-Verlag, Berlin-Heidelberg-New York (1972).)

[8] A. Debiard, B. Gaveau et E. Mazet, "Théorèmes de comparaison en géométrie riemannienne". Publ. RIMS, Kyoto Univ. 12, (1976) $391-425$.

[9] K. Ichihara, "Curvature, geodesics and the Brownian motion on a Riemannian manifold". Preprint, Dept. of Applied Sciences, Faculty of Engineering, Kyushu Univ.

[10] N. Ikeda and S. Watanabe, Stochastic differential equations and diffusion processes. (North Holland Mathematical Library 24 North Holland Publ. Comp., Amsterdam-Oxford-New York, and Kodansha Ltd., Tokyo (1981).)

[11] K. Itô, "On stochastic differential equations". Mem. Amer. Math. Soc. 4 (1951). 
[12] I. Johnstone, A probabilistic study of Zinear elliptic-parabolic equations of second order. (Notes on Pure Math. 12 Aust. Nat. univ., Canberra (1979).)

[13] G. Leha and G. Ritter, "On diffusion processes and their semigroups in Hilbert spaces with application to interacting stochastic systems". Ann. of Probability (to appear).

[14] P. Malliavin, Geometrie differentielle stochastique. (Les Presses de l'Université de Montréal, Montxéal (1978).)

[15] H.P. McKean, Stochastic integrals. (Academic Press, New YorkLondon (1969) .)

[16] P.A. Meyer, Probability and potentials. (Blaisdell Publ. Co., Waltham, Mass.-Toronto-London (1966).)

[17] M. Pinsky, Stochastic Riemannian geometry. In: Probabilistic analysis and related topies, Vol. I, (led. by A.T. BaruchaReid), 199-236. Academic Press, New York-San FranciscoLondon (1978) .)

[18] P. Priouret, Processus de diffusion et equations differentielles stochastiques. In: Ecole d'ete de probabilites de saint Flour III - 1973. ((ed. par P.A. Meyer, P. Priouret, F. Spitzer). Lecture Notes in Math. 390, 37-113 (1974).)

[19] P. Priouret, "Processus de diffusion". Gazette des mathematiciens Soc. math. de France 4 (1975) 71-87.

[20] D.W. Stroock and S.R.S. Varadhan, "Diffusion processes with continuous coefficients I,II. Comm. Pure Appl. Math. 22 (1969) $345-400$ and $479-530$.

[21] D.W. Stroock and S.R.S. Varadhan, Multidimensional diffusion processes. (Grundlehren der math. Wiss. 233, Springer-Verlag Berlin-Heidelberg-New York (1979).)

[22] R. Walter, "Konvexität in riemannschen Mannigfaltigkeiten". Jber. d. Dt. Math.-Verein. 83 (1981) 1-31.

[23] D. Williams, Diffusions, Markov processes, and martingales, Vol. 1 (J. Wiley \& Sons, Chichester-New York-Brisbane-Toronto (1979).) 
[24] D. Williams, "To begin at the beginning:..." In: Stochastic Integrals, (Proc. IMS Durham Symposium 1980 (ed. by D. Williams). Lecture Notes in Math. 851, 1-55 (1981).)

[25] S.T. Yau, "On the heat kernel of a complete Riemannian manifold". J. Math. Pures App Z. 57, (1973) 191-201.

Mathematisches Institut

der Universität Erlangen-Nürnberg

Bismarckstrasse $1 \quad 1 / 2$

8520 Erlangen

Federal Republic of Germany. 